\title{
La scène du bal en littérature : le langage silencieux des émotions
}

Résumé: L'article propose une réflexion sur les possibilités du texte littéraire d'exprimer les émotions. À cet effet, on effectue une analyse comparative de l'épisode $\mathrm{du}$ bal, topos narratif présent dans trois grands romans d'amour : La Princesse de Clèves de Madame de La Fayette, Anna Karénine de Léon Tolstoï et Le Ravissement de Lol V. Stein de Marguerite Duras. Les différences dans la description des trois scènes de bal révèlent différents modèles d'expression de la subjectivité et des variations au niveau de l'émotion amoureuse. Il est donc possible de dire que la ligne de démarcation passe par le degré d'amuïssement du langage, d'une part, et par l'évanouissement de l'Éros, d'autre part.

Mots clés : Langue, silence, émotions, langage du corps, scène du bal

\section{Introduction}

Nous nous proposons de réfléchir sur le langage des émotions à partir d'une lecture comparative de la scène du bal, topos narratif, dans trois grands romans de la littérature amoureuse, à savoir La Princesse de Clèves, Anna Karénine et Le Ravissement de Lol V. Stein. La comparaison implique l'épisode du surgissement de la passion amoureuse lors du bal, afin de mettre en relief des consonances et des dissonances, des variantes et des constantes possibles au niveau du thème et de l'expression littéraire chez les trois auteurs.

Dire que le langage est incapable de transmettre nos émotions est un truisme. Pourtant, la littérature étant l'empire des mots, il n'est certainement pas sans intérêt de voir, dans un premier temps, quels sont les moyens que la langue possède pour exprimer et transmettre l'inexprimable de la passion amoureuse naissante. Prenant en considération les trois textes étudiés, il devient évident que le récit de la scène du bal mobilise des procédés stylistiques et rhétoriques, mais aussi des moyens syntaxiques pour faire face à la déficience du verbal.

Par ailleurs, le texte romanesque met en œuvre divers topos narratifs pour raconter (exprimer et transmettre) les émotions. Le bal en est un. Le récit du bal dans les romans en question introduit le langage du corps, de la danse et des couleurs, qui montre la passion amoureuse naissante. Le verbal (explication, analyse psychologique) se retire, semble-t-il, pour céder la place au non-verbal, notamment le mouvement, les figures de danse, le cri, les larmes, le regard, le soupir... 
Ainsi, divers moyens et procédés sont susceptibles de remédier à l'insuffisance du langage en ouvrant un espace au silence où l'émotion s'exprime par la tension de l'inexprimé que le langage du corps laisse deviner. L'étude comparative permettra de voir comment cet espace est construit dans chacun des romans.

Dans un deuxième temps, la réflexion porte sur l'évolution des relations entre subjectivité et société. Les trois scènes de bal mettent en valeur le moment du surgissement de la passion amoureuse et témoignent des diverses manières de vivre et d'exprimer cette passion dans une société. Comment le rapport entre l'amour et la société change-t-il ? Le rapport au silence, en tant que retrait du langage, représente-t-il une ligne continue qui relie le roman galant, le roman réaliste psychologique et le roman post-moderne?

\section{2 Émotion, sentiment, passion}

Au cours de la scène du bal dans les trois romans, les protagonistes vivent l'effet de la passion naissante et se trouvent en proie à un complexe émotionnel difficile à démêler. Il semble donc important de rappeler certaines différences entre les notions d'émotion, de sentiment et de passion.

Tout d'abord, les protagonistes éprouvent des émotions très fortes, résultat d'un effet de séduction irrésistible. Or l'émotion est un état psychique spontané et peu durable qui entraîne des réactions physiques : pleurs, cris, rougissement, évanouissement. Lol s’immobilise puis crie, Kitty retient mal ses larmes.

Selon Sartre les psychologues admettent que « l'émotion est d'abord et par principe un accident » $(1995,14)$.

En effet, dans les romans étudiés, l'amour qui advient fortuitement au cours du bal, sans aucun signe précurseur, possède le caractère d'un accident. C'est l'accident de l'amour.

Les émotions peuvent prendre la forme plus concrète de sentiments. Pendant le bal, les personnages connaissent la surprise, l'éblouissement, la confusion, l'incertitude, la douleur. Tous ces sentiments existent simultanément, ou bien le personnage passe d'un état à l'autre.

Mais les personnages des trois scènes sont surtout en proie à la passion. Étymologiquement, passion vient du latin patior, pati, dont l'équivalent grec est pathos. Au sens philosophique, passion signifie souffrance, supplice. Il est important de souligner que c'est un état de celui qui subit, un état de passivité. La volonté est passive, la raison aussi, face aux impulsions du corps. Pensons au roman de Duras où, lors du bal, Anne-Marie Stretter, une fois dans les bras de Michael Richardson, a une « expression abêtie, figée par la rapidité du coup » 
(Duras 1964, 19). Au sens moderne la passion est une forte inclination pour un objet, un état affectif durable, ce qui entraîne un déséquilibre psychologique.

Nous nous demanderons donc comment ce déséquilibre est exprimé par des moyens langagiers.

\section{Langage et émotion}

Platon n'a-t-il pas dit que l'immensité de l'être anéantit la parole ? Le langage impose des taxinomies, charrie des significations consensuelles, bloque l'envol de la pensée et s'évanouit devant l'accident des émotions. Le langage semble fatigué.

Mais il possèderait la capacité de produire des textes amuïs pour échapper à ses propres limites en préparant l'avènement du silence, une forme radicale de l'indicible, de l'inexprimable et de l'inavouable. Le texte narratif ou dialogique mobilise divers mécanismes et procédés stylistiques pour rendre tangible le silence éloquent. Par exemple, l'exclamation qui emporte la parole, l'aphasie qui anéantit la réaction verbale, mais aussi les figures rhétoriques comme l'ellipse qui suggère le non-avouable et la répétition qui peut marquer un dire impossible ou un dire insistant. Sans oublier le rythme de la phrase, marqué par le suspense et la reprise.

Le propre de la littérature n'est-il pas de passer outre la médiation langagière, de construire un texte « imparfait » pour ouvrir l'espace d'un après-texte où le lecteur soit libre d'imaginer une infinité de sens investie dans le non-dit, l'inexprimé ou l'inavoué ? Dans le cas des bals, le récit donne à voir l'accident de l'amour : regarder et voir viennent remplacer avouer et expliquer. Le texte dit moins qu'il ne signifie.

Comment exprimer l'émotion sans la dire ? " Le mystique en transe n'écrit pas » (Michaux 1998, 960). Rappelons-nous le fameux billet de Pascal, écrit sous l'effet de l'extase.

D’autres codes sémiotiques entrent en jeu pour suppléer aux insuffisances du langage à transmettre les passions : la musique, les couleurs, la gestuelle, le corps. Mais la sémiotique non verbale en est encore à ses débuts. Ainsi, selon Grigorij Krejdlin, " il est nécessaire de trouver une approche sémiotique unique pour étudier les moyens verbaux et non-verbaux dans la communication » (Krejdlin 2007, 2).

Dans les trois scènes de bal, c'est le langage corporel qui nous intéresse. Il comprend la gestuelle, l'expression du visage, le regard, les vêtements (couleurs, coupes, tissus), les mouvements de la danse, la coiffure. Le texte confère au lecteur le rôle de spectateur. 
L'étude comparative de l'épisode du bal portera sur les diverses formes d'engagement de ces codes sémiotiques autres que le langage pour élaborer le spectacle de l'avènement de l'amour.

Par ailleurs, l'épisode du bal peut être étudié dans le contexte de la communication sociale qui englobe plusieurs facteurs : les participants, danseurs et spectateurs ; le caractère de la danse, l'ambiance, le mobilier. La description de ces facteurs par le récit contribue à compléter le spectacle et à mettre en relief l'état émotionnel des protagonistes.

\section{Le bal dans les trois romans}

Les trois ouvrages où l'épisode du bal possède une fonction matricielle, mais aussi structurante, font partie des romans d'amour dits classiques.

La scène du bal est construite à partir de stéréotypes culturels qui opèrent à des niveaux différents, tels que :

- La structure actancielle : le triangle amoureux, comprenant deux actants et un troisième qui est dans un rôle d'observateur, éventuellement le tiers absent. Dans La Princesse de Clèves, ce troisième personnage est implicite : le prince de Clèves n'est présent que par le nom de la princesse. Le chevalier de Guise est un substitut du tiers exclu.

- Le niveau thématique : ce qui rapproche les trois scènes, c'est le thème de l'amour naissant et de l'abandon, mais aussi le motif de la prédestination. En fait, les protagonistes se sont croisés avant le bal. Il y a donc une pré-rencontre. À l'exception d'Anna Karénine, où les trois protagonistes se connaissent vraiment, dans les deux autres romans, ils se sont seulement entrevus par hasard (Le Ravissement de Lol V. Stein), ou se connaissent comme membres de la même société (La Princesse de Clèves).

- Le schéma diégétique, presque identique, que le récit du bal suit dans les trois textes : l'entrée en scène des protagonistes, la musique, la danse, les réactions des spectateurs, le moment de la rencontre, l'abandon du tiers. Dans les trois épisodes il y a aussi une mise en place : la description du lieu, du public.

- La fonction narrative du bal est déstructurante/restructurante (Montandon 1998, 14) : quelque chose du passé des protagonistes est détruit et quelque chose de nouveau est annoncé. Au niveau diégétique, le bal joue le rôle d'une prolepse. 
Mais ces stéréotypes sont différemment explorés dans les trois textes.

Une première différence s’impose : dans La Princesse de Clèves, le bal est raconté selon les règles du style galant ; dans Anna Karénine, on a affaire à une description réaliste et au récit suggestif psychologique des émotions : dans Lol $V$. Stein, c'est par le récit minimaliste et hésitant que l'auteure efface le dire au profit d'un silence éloquent.

Une deuxième différence : dans le roman de Madame de la Fayette, la rencontre des deux protagonistes semble provoquée par le roi. C'est une « rencontre programmée ». C'est la cour qui autorise la rencontre et c'est sous les yeux de la cour que la passion amoureuse évolue ; chez Tolstoï, le surgissement de l'amour est une aventure personnelle sous les yeux du public ; chez Duras, apparemment, les mêmes stéréotypes du bal d'amour sont en jeu. Ne dit-elle pas qu'elle écrit ses livres « avec les autres » ? Mais après être passé par la « chambre hallucinatoire de l'écriture », le texte change. La réécriture intertextuelle que Duras opère s'obstine à défaire les stéréotypes. Dans ce cas concret, cela passe, selon Sylvie Bourgeois, par la déconstruction et l'amplification. La passion amoureuse est une passion mortelle. Anne-Marie Stretter n'est pas seulement la femme fatale, elle est l'incarnation de la mort. Le dépérissement de Lol est un traumatisme psychologique. Le récit de l'épisode est incertain et incomplet. Il s'achève dans la stupéfaction et dans l'incompréhension de l'évènement.

\section{Comment dire la passion naissante ?}

Dans les trois romans, l'accident de la séduction est transmis par l'amuïssement de la langue. Les trois textes mettent en œuvre des moyens et des procédés différents pour élaborer cet effet.

\section{La Princesse de Clèves : l'entre-deux de la langue galante}

$\mathrm{Au} \mathrm{XVII}{ }^{\mathrm{e}}$ siècle la société n'est plus régie essentiellement par une force extérieure, le Dieu tout puissant, mais aussi par des individus libres pour lesquels le bon commerce est de première importance. La vie sociale est réglée par des relations dichotomiques telles que : marque et effacement, privé et public, présence et absence, intériorité et extériorité, silence et paroles.

La Princesse de Clèves de Madame de La Fayette est un des romans les plus brillants de la littérature précieuse. Le discours galant est marqué par le souci de dire sans dire. Le code de la préciosité ne tolère pas le directement intelligible. 
À cette époque, les premières danses à deux apparaissent déjà, mais les couples restent ouverts et la répartition des danseurs obéit à des règles strictes. Le bal dont il s'agit dans le roman est organisé par la haute aristocratie.

L'épisode représente le surgissement de la passion amoureuse entre le duc de Nemours et Madame de Clèves sous le contrôle du roi et sous les yeux d'une société d'aristocrates. Les deux protagonistes ne se parlent même pas directement, mais par l'intermédiaire de la Dauphine. Pour Jean Rousset, c'est une « communication entravée » (Rousset 1984, 104). L'émotion qui gagne les protagonistes quand ils se voient est subite, immédiate et indéfinissable. L'auteure désigne cet état émotif par les sentiments de « surprise et d'étonnement ». La répétition de ces vocables (surprise, étonnement, grand étonnement) qui abondent dans le récit sert à transmettre la difficulté de la langue de nommer l'intensité d'un état psychique, advenu brusquement, mais ayant une grande importance. En outre, par la répétition, le récit insiste sur cette difficulté et cette importance.

Lors du bal, l'étonnement chez Madame de Clèves fait place à « l'embarras » et chez le prince à « des marques d'admiration » qu'il ne réussit pas à cacher. Cependant, le texte ne désigne pas ces marques. Du côté du couple regardé, les sentiments sont donc provoqués par quelque chose qui n'est pas de l'ordre du commun et qui n'est pas exprimé dans le récit. Du côté des regardants, le roi et les reines, c'est aussi un sentiment d'admiration qui surgit, mais il est désigné par le récit comme « un murmure de louanges ». Le vocable « murmure» signifie la retenue de l'émotion de la part du public. La perception qui résume cette première scène de la rencontre des deux protagonistes est qualifiée de « singulière ». Mais qu'est-ce qui est singulier ? C'est l'harmonie parfaite du couple des deux danseurs, alors qu'apparemment ils ne se connaissent pas. Suivant le code de la galanterie, l'auteure élabore un récit indécis de la première scène de la rencontre en se servant d'un vocabulaire vague pour signifier le premier émoi sans le dire.

En présence de la Dauphine, le jeu de la séduction passe par un échange de répliques ambiguës sur l'identité des protagonistes : se connaissent-ils ou pas ? Le prince admet qu'il connaît Madame de Clèves, mais quant à elle, elle n’avoue pas qu'elle le connaît sans l'avoir jamais vu. Avant l'épisode du bal, le texte précise qu'elle « avait ouï parler de ce prince », et que « Madame la Dauphine le lui avait dépeint d'une sorte... » (Madame de La Fayette 1999, 71). Cet échange de propos complaisants est nécessaire pour dissimuler la passion naissante entre les deux.

Les pronoms indéfinis tels que quelqu'un, quelque chose sont répétés plusieurs fois. La séduction est un phénomène qui provoque une émotion impossible à nommer et à expliquer. C'est le quelque chose qui engendre le « je-ne-sais-quoi » du récit. Le lecteur en est bouleversé parce qu'il subit la tension d'une passion indicible. 
Anna Karénine : la description du bal et la suggestion des émotions

Chez Tolstoï, le bal se déroule à une époque où la valse est déjà à la mode, du moins dans la haute société. À la différence des danses collectives, qui continuent d'ailleurs à avoir du succès, la valse permet une plus grande intimité, la séduction devient un moment important. Le langage du corps (mouvement, regard, vêtements) retrouve toute sa valeur. Les relations de genre sont codifiées.

La description du bal, spectacle riche et brillant, est une preuve de la maîtrise de Tolstoï. Suivant la tradition du romantisme russe lors de sa transition vers le réalisme, l'auteur n'omet aucun détail du spectacle du raffinement et de l'aisance du beau monde : les invités, hommes et femmes de la haute société, les groupes des jeunes et celui de leurs aînés et de leurs parents, le groupe des valets, l'élégance des robes et des coiffures, la décoration de l'espace.

$\mathrm{Au}$ centre de ce tableau se trouve la figure de Kitty. Le récit suit l'évolution de son état affectif qui passe d'une exaltation heureuse, d'une confiance en sa beauté et en son charme, à la confusion, à une anxiété grandissante, à une horrible angoisse. Ces sentiments sont désignés explicitement dans le texte. Mais la cause de l'état de Kitty n'y est pas mentionnée. Le récit se sert de divers procédés pour ne pas expliquer la raison de l'abattement de la jeune fille, pour retarder le moment de la découverte inattendue.

D’abord, les conversations au style direct sont des échanges de répliques banales, galantes, souvent à bâtons rompus. Les trois protagonistes ne se disent rien de précis. Mais la banalité des propos, le bavardage, cache quelque chose d'important qui s'annonce et qu'il est difficile d'avouer.

Ensuite, les trois points de suspension, le point d'interrogation, l'exclamation, bref la ponctuation construit l'image de la difficulté de l'échange verbal.

En outre, Kitty n'ose même pas nommer Vronski dans son discours intérieur. Elle le désigne par « lui ». Est suggérée ainsi la force de l'émotion qu'elle éprouve quand elle le voit, quand elle l'observe. Cette émotion fait peur, l'objet de cette émotion ne doit pas être nommé, il ne peut être nommé. Nommer, c'est faire exister. Or, Vronski est intimement proche et en même temps, il provoque un sentiment de crainte si fort chez Kitty qu'il doit rester loin, confiné dans ce pronom de la troisième personne, « lui », qui correspond, selon Benveniste, au mode de la non-personne.

Enfin, la répétition du vocable séduction, mais aussi séduisant/e, est significative : " séduisante était sa robe, séduisants ses beaux bras, séduisant son cou, [...] » (Tolstoï 1952, 96). Kitty est fascinée et hantée par l'effet de séduction que provoque Anna.

Le fragment où l'adjectif séduisant est répété six fois culmine par deux qualificatifs de sens très fort, notamment terrible et cruel, mais associés à un objet imprécis, quelque chose : « il y avait dans cette séduction quelque chose de terrible 
et de cruel » (Tolstoï 1952, 96). Ce « quelque chose » paraît en dehors de nos capacités d'appréhension et de verbalisation.

Le narrateur construit donc son récit dans un régime d'alternance entre dire et taire pour suggérer l'évènement inespéré qui est en train de se produire.

\section{Le Ravissement de Lol V. Stein : le langage silencieux des émotions}

Pendant les années 1960 où Duras écrit son roman, le bal est un des loisirs qui caractérisent une société en plein essor économique et une classe moyenne qui s’affirme par le plaisir : congés payés, voyages, nourriture, musique. Lors du bal, la joie collective prend forme, les rencontres ont facilement lieu, le corps se libère dans la danse, les couples se font et se défont, les mouvements s'improvisent. Les relations de genre ne semblent pas affectées par les contraintes du code.

Le récit du bal dans Le Ravissement se met en question dès la première page. Le métarécit annonce la déconstruction du récit.

L'épisode du bal commence in media res, la mise en place est très brève. Le lecteur est introduit dans le spectacle et incité à sentir l'ambiance à partir de deux petits paragraphes de construction identique. Arrêtons-nous sur le deuxième :

La piste s'était vidée lentement. Elle fut vide. (Duras 1964, 15)

L'extrême dépouillement des deux phrases suscite la perception d'un évènement insolite. La première présente l'action comme un processus dont l'accomplissement est caractérisé par l'adverbe lentement qui aide à visualiser le mouvement. La deuxième phrase, en régime de juxtaposition, offre le résultat du processus. L'accomplissement de l'action comme processus, donné au plus-queparfait, présuppose l'accompli, donné au passé simple. Pourquoi le récit insistet-il sur le résultat? Pourquoi la répétition du même vocable sous ses deux formes, vider/vide ? N'est-ce pas pour perturber la lecture linéaire et suggérer quelque chose d'inattendu qui va se produire sur cette piste désertée ? Par ailleurs, l'économie des deux phrases contribue à intensifier l'attente du lecteur qui devient spectateur. Ainsi, la compréhension évolue au sein d'une expression linéaire minime qui construit un spectacle dont le sens est pressenti dans sa verticalité profonde, inexprimée et inexprimable. L'étrange apparition d’Anne-Marie Stretter est inexprimable.

La simplicité de la langue dans laquelle les quelques évènements de la soirée sont racontés est sans cesse dérangée par l'intrusion de mots de sens général, abstrait et absolu, qui se détachent des phrases et creusent des lacunes de sens dans le tissu textuel. La disjonction (concret, simple vs. abstrait, absolu) qui se produit oriente la perception vers d'autres niveaux de sens en latence, transcendant le récit des faits et provoquant un effet de silence, où une réalité différente est pressentie dans l'après-langue : « les emblèmes d'une obscure négation de la nature ; son élégance [...] inquiétait » (Duras 1964, 15) ; « cet âge [...] dans les fous, 
endormi », « cet autre aspect des choses » (Duras 1964, 20) ; " elle guettait l'évènement, couvait son immensité » (Duras 1964, 18) ; "quelque signe d'éternité » (Duras 1964, 21). La tension irrésolue, élaborée ainsi, est renforcée à l'aide du mutisme des protagonistes, directement indiqué par les formes négatives de dire et indirectement par les injures de la mère, la modulation plaintive et le cri de Lol.

Le langage construit donc un effet de silence qui engendre le pressentiment d'une fatalité imminente : « aucun mot [...] n'aurait eu raison du changement de Michael Richardson » (Duras 1964, 17), tandis que Lol ne peut que sourire.

En fait, c'est Duras qui est allée le plus loin dans l'élaboration de la technique du dire sans dire pour narrer des histoires où les mots précipitent le sens dans le silence de l'inexprimable. Mais ce silence est imparfait, car il provoque une tension du sens qui incite le lecteur à sentir l'extase, le désir, la passion.

\section{Le langage du corps}

Le corps est le premier émetteur de signes lors de la communication humaine. Le récit du bal engage le lecteur à déchiffrer ces signes et à visualiser le spectacle de la rencontre. Le corps participe donc pleinement au spectacle du bal.

\section{La Princesse de Clèves : le charme deviné}

Le récit introduit le prince dans la scène du bal d'abord par le bruit qu'il produit en entrant dans la salle. Ainsi, le lecteur entend l'arrivée du prince de Nemours et il voit le prince en même temps que la princesse de Clèves le voit. Mais l'arrivée du prince en scène se fait d'une manière insolite : il passe par-dessus quelques sièges. De la sorte, le récit donne à voir l'aspect physique du prince : jeune et agile. Cette performance montre les qualités physiques du personnage et suggère un caractère impatient, intrépide et libre par rapport à l'étiquette. Le récit du corps promet un déroulement inattendu.

Plus loin, le corps parle à travers la révérence que fait la princesse et qui n'est nullement décrite. Le récit fait valoir ce mouvement par la réaction de Monsieur de Nemours ne pouvant pas s'empêcher de donner des marques d'admiration qui ne sont toutefois pas précisées. Le lecteur doit imaginer la grâce de la révérence qui caractérise indirectement la princesse : jeune, naturelle, innocente, modeste, élégante. À la hardiesse de Monsieur de Nemours correspond donc la délicatesse de la princesse. Le récit du langage corporel prépare le je-ne-sais-quoi de la séduction amoureuse qui contient le secret de multiples plaisirs. 
Lors de la danse, les corps et les mouvements ne sont pas décrits. C'est par le murmure de louanges dans la salle, donc une image auditive, que le récit témoigne de leur beauté.

L’arrivée du prince, la révérence et la danse sont des moments du spectacle du bal qui mettent en évidence la grâce des personnages. Cette grâce qui déclenche la séduction n'est pas transmise par le dit, mais par ce qui est entendu, vu et regardé, que la narration élabore. Ainsi, rien n'est dit directement, c'est l'imagination auditive et visuelle du lecteur qui est stimulée par le texte. Le lecteur interprète plutôt ce que le texte lui donne à entendre et à voir que ce qu'il lui explique. Le récit de l'épisode du bal suit les principes de l'art de plaire mondain, qui est une pratique et une esthétique. Le charme est vu, sans être dit.

\section{Anna Karénine : l'éloquence du langage du corps}

Le récit du corps commence par l'arrivée au bal de Kitty et de sa mère.

Le premier paragraphe ne décrit pas Kitty directement. Sa beauté est suggérée par les réactions des autres personnages présents dans l'épisode. Le même procédé est en œuvre dans La Princesse de Clèves, où le récit signifie indirectement la grâce de monsieur de Nemours et de la princesse de Clèves.

Suivent plusieurs paragraphes où le texte engage le récit de l'apparence extérieure de Kitty : la robe, la coiffure, les bijoux, le ruban. Le luxe de la toilette de la jeune fille a un effet érotique. L’aisance du corps est signifiée par le naturel de la toilette. La couleur rose de la robe, les petits pieds chaussés de rose, mais aussi les lèvres roses, viennent signifier l'innocence joyeuse de la jeune fille et la confiance en son triomphe. Plus loin cette vague de rose va être opposée aux couleurs sombres (velours noirs) de la robe d'Anna. L'insouciance, la joie, la gaieté de Kitty sont donc mises en évidence indirectement par la description que le récit fait de sa toilette de bal. Couleurs, tissus, coupes parlent le langage de l'élégance naturelle d'un être qui respire le bonheur de l'amour.

Les signes que la toilette de Kitty (et le corps indirectement) disponibilise viennent contraster avec ceux de la toilette d'Anna. D’abord, au niveau des couleurs : le rose de l'insouciance et le noir du malheur incontournable. Ensuite, au niveau des tissus : la légèreté de la robe de Kitty, le tulle rose, et le poids du velours de la robe d'Anna. De plus, la haute coiffure de Kitty et la coiffure fort simple d'Anna. L'unique similitude, c'est le naturel avec lequel les deux protagonistes portent chacune sa toilette.

Tout le récit de l'apparence extérieure suggère la différence entre Kitty et Anna et prépare le lecteur à la perception d'un déroulement insolite de l'histoire.

La danse, c'est-à-dire les pas, les figures, les mouvements du corps, les gestes, les regards échangés, l'expression des visages, annonce le malheur de Kitty. La jeune fille ne fait que « quelques pas de valse » avec Vronski qui ne lui tient que 
« des propos insignifiants » (Tolstoï 1952, 93). Le récit donne à voir la confusion de Kitty, la perte de confiance, le désarroi : « elle se laissa tomber »; " un de ses bras nus, maigre et délicat, retombait sans force »; « visage brûlant » (Tolstoï 1952, 95). C’est pendant le dernier quadrille que la révélation a lieu. D’abord, le récit transmet le triomphe d’Anna : « le regard enflammé ; le sourire de triomphe ; les lèvres entrouvertes ; la grâce et l'harmonie suprême des mouvements » (Tolstoï 1952, 94). Ensuite vient le moment culminant de la révélation : Kitty découvre la réciprocité de l'émotion sur le visage de Vronski et sur celui d’Anna : « elle le regarda et fut épouvantée, car le visage de Vronski reflétait comme un miroir l'exaltation qu'elle venait de lire sur celui d'Anna » (Tolstoï 1952, 94). La grande émotion chez Vronski se manifeste, outre l'exaltation qui correspond à celle d'Anna, par l'angoisse et la soumission que l'on lit dans son regard, " la tête baissée, prêt à se prosterner », ce qui représente un changement par rapport à son « maintien résolu et à sa physionomie toujours en repos » (Tolstoï 1952, 94) que Kitty lui connaît.

L'éloquence du corps dans le roman de Tolstoï est d'une force évidente : dansant ou immobile, frêle ou ferme, hésitant ou résolu, meurtri ou exalté, c'est le récit du corps qui élabore le spectacle de la séduction et de l'abandon.

\section{Le Ravissement de Lol V. Stein : le corps qui parle l'amour-mort}

Le corps d'Anne-Marie occupe le récit dès le début. Mais l'accent ne tombe pas sur la toilette, seuls sont mentionnés quelques éléments stéréotypés comme : la robe noire, très décolletée, un fourreau de tulle également noir. C'est au corps physique que le récit s'intéresse et ce corps parle le langage d'une élégance étrange et perturbante. Nous lisons : taille haute, charpente un peu dure, ossature admirable, décoloration pénible de la pupille, regard difficile à capter, rousseur, Ève marine. Le stéréotype de la beauté et de la délicatesse féminine est détourné. La séduction passe par la sensation de bizarrerie, de surprise inquiétante.

Le récit du corps d'Anne-Marie donne à voir la scène de rencontre comme un accident tragique. Au spectacle de ce corps, Michael Richardson subit des changements, repérés par les autres participants à la scène : « les yeux s'étaient éclaircis », « son visage s’était resserré [...] » (Duras 1964, 17). À la fin du bal, « tous les trois, ils avaient pris de l'âge [...] des centaines d'années, de cet âge, dans les fous, endormi » (Duras 1964, 19-20).

L'accident de la séduction se produit avant même la rencontre physique dans la danse et transforme les personnages en marionnettes : Lol est « frappée d'immobilité », Michael Richardson " pâlit », " comme des automates, ils [Anne-Marie Stretter et Michael Richardson] s'étaient rejoints » (Duras 1964, 21). Leurs mouvements automatiques appellent le silence qui signifie leur souffrance. C'est une souffrance qui rejoint l'immensité du vide, Lol oublie de souffrir. 
Le bal d'amour chez Duras est un spectacle de séduction mortelle. Le récit entraîne le lecteur dans la scène, il devient participant et spectateur.

\section{Conclusion}

La comparaison de la scène du bal dans les trois romans, effectuée à partir de l'analyse du langage des émotions, est un modeste effort pour compléter les études critiques où, le plus souvent, ce sont l'aspect thématique des émotions et la fonction diégétique de l'épisode en question qui sont abordés. On renverra par exemple à l'article de Jean-Marie Schaeffer et Ioana Vultur, intitulé Fictions autophages chez Marguerite Duras, qui étudie le caractère matriciel de la scène du bal dans les romans du cycle indien de l'écrivaine.

L'analyse des mécanismes et des formes de l'amuïssement du langage, qui prépare lui-même et valorise son échec à exprimer les passions, permet d'esquisser trois régimes de silence qui marquent la différence entre les trois épisodes : dans La Princesse de Clèves, c'est le silence de la langue de la galanterie ; dans Anna Karénine, c'est le silence de l'inexplicable, de l'inavouable de la séduction ; dans Le Ravissement de Lol V. Stein, c'est le silence de l'indicible face à la fatalité du hasard. En outre, les nombreux signes du corps mobilisés par le récit contribuent à « vivre » le spectacle du bal dans la tension muette du regard.

Par ailleurs, l'analyse comparative a permis de voir comment les stéréotypes de la séduction amoureuse changent, tout en préservant le rôle de « l'éternel féminin » : du secret de la féminité et de la délicatesse de la femme dans La Princesse de Clèves, on passe par l'attrait démoniaque d’Anna dans Anna Karénine pour arriver à la tentation mortelle d'Anne-Marie Stretter dans Le Ravissement. La passion naissante lors de la rencontre adopte aussi des formes différentes : une passion " programmée » dans La Princesse de Clèves ; une passion « terrible et cruelle » dans Anna Karénine ; une passion fatale dans Le Ravissement de Lol V. Stein.

La valeur érotique de la rencontre amoureuse change progressivement. Le trouble du bonheur et la beauté de la révélation amoureuse dans la Princesse de Clèves se transforment en un désir périlleux dans Anna Karénine, pour devenir un désir mortel dans Le Ravissement.

De nos jours, la joie, la séduction cessent-elles d'assurer le bonheur du couple ? L'amour est profané de telle façon qu'il se transforme de plus en plus en accident sexuel. Selon Alain Badiou, la « menace sécuritaire » détruit le bonheur de l'aventure amoureuse et l'excitation devant le hasard et le risque. Aujourd'hui, c'est le plaisir du corps (epithymia), semble-t-il, qui domine l'expérience du désir 
de l'âme. Le romantisme de la rencontre amoureuse, représentée par de grandes œuvres littéraires, pourrait-il encore servir d'antidote à cet amour qui craint l'accident des sentiments et l'échec des émotions, afin d' "Essayer quoi ? Vous dites : D’aimer » (Duras 1982, 9) ?

\section{Bibliographie}

Badiou, Alain, avec Nicolas Truong. Éloge de l'amour. Paris : Flammarion, 2009.

Bourgeois, Sylvie. «Le bal du Ravissement de Lol V. Stein : une réécriture subversive de deux grandes scènes de bal stéréotypées ». Les lectures de Marguerite Duras. Alexandra Saemmer et Stéphane Patrice (dir.). Paris : PUL, 2005. 35-43.

Buffault, Anne-Vincent. Sensibilité et insensibilité : des larmes à l'indifférence. http://www. fabula.org/colloques/document2322.php. (19 juin 2018).

Coudreuse, Anne. « La rhétorique des larmes dans la littérature du XVIII e siècle : études de quelques exemples ». Modèles linguistiques [En ligne] 58 (2008). http://journals. openedition.org/ml/379. Mis en ligne le 11 septembre 2013. (15 août 2018).

Duras, Marguerite. Le Ravissement de Lol V. Stein. Paris : Gallimard, « folio », 1964.

Duras, Marguerite. La Maladie de la mort. Paris : Les Éditions de Minuit, 1982.

Dziub, Nikol. « Histoire du silence à l'âge moderne ». Acta fabula vol. 18, no 2 (février 2017). http://recherche.fabula.org/revue/document10063.php. (19 juin 2018).

Han, Byung-Chul. A Agonia de Eros. Miguel Serras Pereira (trad.). Lisbonne : Relógio D’Água, 2014. [Original all. : Agonie des Eros. Berlin : Matthes \& Seitz, 2012].

Krejdlin, Grigorij. « Le langage du corps et la gestuelle ». Françoise Daucé (trad. du russe). UFR d’Études slaves, Université de Paris-Sorbonne. Cahiers slaves no 9 (2007) : 1-23.

La Fayette, [Marie-Madeleine, dite] Madame de. La Princesse de Clèves. Paris : Le livre de poche, « Classique », 1999.

Méchoulan, Éric. Le corps imprimé : essai sur le silence en littérature. Montréal : Balzac-Le Griot, 1999.

Michaux, Henri. «Absence » [1934]. Euvres complètes, t. 1. Paris : Gallimard, « Bibliothèque de la Pléiade », 1998.

Montandon, Alain. « Pour une sociopoétique du chronotope : la scène de bal chez Théophile Gautier ». Littérature vol. 112, n 4 (1998) : 14-25.

Rousset, Jean. Leurs yeux se rencontrèrent, la scène de première vue dans le roman. Paris : Corti, 1984.

Sartre, Jean-Paul. Esquisse d'une théorie des émotions. Paris : Hermann, 1995 [1938].

Schaeffer, Jean-Marie, et loana Vultur. « Fictions autophages chez Marguerite Duras. À propos du cycle indien ». Poétique 1, nº 145 (2006) : 3-24.

Tolstoï, Léon. Anna Karénine. Paris : Gallimard, « folio classique », 1952. 
Zlatka Timenova-Valtcheva was born in Sofia, Bulgaria, in 1949. In 1979 she received a $\mathrm{Ph}$.D. from the University of Sofia with her thesis La métaphore dans Les Pensées de Blaise Pascal. In 2008 she received a Doctorate in Modern Languages and Literatures from the University of Coimbra, Portugal. The thesis was submitted under the title Le silence et ses formes dans l'œuvre romanesque de Marguerite Duras. She is currently teaching French language and Bulgarian language and literature at the University of Lisbon. Her research interests include the theory of literature, translation theory, and comparative literature. Z. Timenova-Valtcheva is a member of the following institutions: WHA (World Haiku Association), ICLA (International Comparative Literature Association), PEN Bulgaria, AFH (Association francophone de haiku). She has published several works of poetry, as well as literary criticism, translations and self-translations. Recent titles include "Slavonic and Iberian perspectives on Silence”. Iberoslavica. Lisbon, 2011, and Silence. Babilonia. Lisbon, 2012. She participated in conferences and seminars in Paris, Vienna, Seoul, Tokyo, Sofia, and Lisbon. In addition, she has authored 4 books of haiku, and published haiku in anthologies and journals in Portuguese, English, French, Bulgarian, Arabic, Japanese, Vietnamese, Croatian, and Italian. She is the winner of the following international contests: IAFOR (2016), and Europoésie (2016 and 2017). 